\title{
A Risk for Nosocomial Infection: Contamination of Hospital Air Cooling Systems by Acanthamoeba spp.
}

\author{
Mahdi Mosayebi ${ }^{*}$, Reza Hajihossein ${ }^{1}$, Behzad Ghorbanzadeh², Saba Kalantari \\ ${ }^{1}$ Department of Parasitology, School of Medicine, Arak University of Medical Sciences, Arak, Iran. ${ }^{2}$ Department of \\ Parasitology, School of Medical Sciences, Tarbiat Modarres University, Tehran, Iran. ${ }^{3}$ Department of Occupational \\ Health Engineering, School of Public Health, Tehran University of Medical Sciences, Tehran, Iran.
}

First Published online March 30, 2016

\begin{abstract}
Background and Objectives: In the most Iranian hospitals the internal air is cooled by a system comprising centrifugal blower fan and excelsior filters. Because amoeba can be present in air and dust it may enter into filters of cooling systems. It has been suggested that diffusion of amoebae cyst through air canals may contribute to various types of nosocomial infections. This study thus aimed to examine the possible contamination of filters of the conventional hospital cooling systems with free-living amoebae (FLA).
\end{abstract}

Methods: Eighty-four cooling systems from four hospitals in Arak city (Central Iran) were sampled. Samples were cultured in defined medium and were tested from presence of Acanthamoeba spp.

Findings: Ten out of the 18 hospital wards/sites and 23 out of 84 cooling systems surveyed (27.3\%) were found to be contaminated by amoebae.

Conclusions: Our results indicate a significant level of amoebae cysts contamination of cooling systems with excelsior filters in various hospital wards. Given the potential contribution of cyst to nosocomial infection, a prompt addressing of the problem is warranted. This may partly be accomplished by using non-aqueous or closed circulation cooling systems. Our study also highlights the need for similar investigations in other health settings and in larger scales to gain insight into the extent of the problem and the associated risks.

Keywords: Nosocomial infection, Patient safety, Air cooling system, Environmental health

\section{Background and Objectives}

Excelsior filters provide a simple and effective method for cooling air of houses and buildings, such as hospitals, in most areas of Iran. When these filters are wet by pouring water that circulate by water pumps, the interlocking fibers in excelsior are well moisturized. Then cooled air is sucked from pores of them and flowed to indoor via canals by centrifugal blower fan. These filters in the outdoor exposed to contamination by dust and many other particles, fungi and microorganisms such as free living amoeba. Free living amoeba completes its life cycle without a host organism in the environment. ${ }^{1}$ They can be found in natural and artificial environments such as water, soils and

*Corresponding Author: Mahdi Mosayebi, Department of Parasitology, College of Medicine, Arak University of Medical Sciences, Arak, Iran. Tel: +98-9108060321; Fax: +98-8634173521; Email: m.mosayebi@arakmu. acir cooling systems. ${ }^{2,3}$ It has been suggested that diffusion of cyst through air canals may contribute to nosocomial infections and result in adverse health effects especially in hospitals' eye, burn, transplant, pediatric (malignancies), HIV, and oncology wards. A group of free-living amoebaes (FLAs) are belonged to the genus Acanthamoeba and are the causative agents of granulomatous amebic encephalitis (GAE), and amebic keratitis (AK). Acanthamoeba spp. also has been associated with cutaneous lesions and sinusitis in patients involved with AIDS and other immunocompromised individuals. ${ }^{4}$ Also FLA has been described as reservoirs for pathogenic bacteria. ${ }^{5}$ Because amoeba can be found in air and dust so it may enter into filters of cooling systems. Therefore, we were motivated to investigate whether the filters of cooling systems with excelsior filter of hospitals in Arak (Central Iran), might be contaminated with Acanthamoeba spp. 


\section{Methods}

This study was conducted in Arak the center of Markazi province situated in Central Iran $(295 \mathrm{~km}$ from to the Tehran the Capital of Iran) (Figure 1). It is situated in area having an elevation of $1703 \mathrm{~m}$ above sea level. The winter months are much rainier than the summer months in Arak. The average temperature in Arak is $10.8^{\circ} \mathrm{C}$. In a year, the average rainfall is $557 \mathrm{~mm}{ }^{6}$

For this study, 168 samples from the total of eighty four cooling systems of four hospitals located in different areas of Arak city were examined. A cross-sectional study using random sampling was conducted during December of 2014 to January of 2015.

The cooling systems were located in outdoor, mostly on the roof of the hospitals. Eighty-four samples were collected by cassettes and a high volume sampling pump (the SKC MCS Flite, USA) and 84 samples were collected by breaking pieces of heavily soiled fibers of excelsior. Microvacuum cassettes were prepared by a stainless steel filter holder and $0.4 \mu \mathrm{m}, 47 \mathrm{~mm}$ diameter polycarbonate filters (Sartorius Stedim Biotech $\mathrm{GmbH}$,

Germany) for sampling of settled dust on excelsior filters in $20 \times 20 \mathrm{~cm}$ foursquare for 4 minutes. Each sample was placed into a sterile polypropylene container and transported to the laboratory for subsequent analysis.

The hospital code, site of cooling system, name of ward

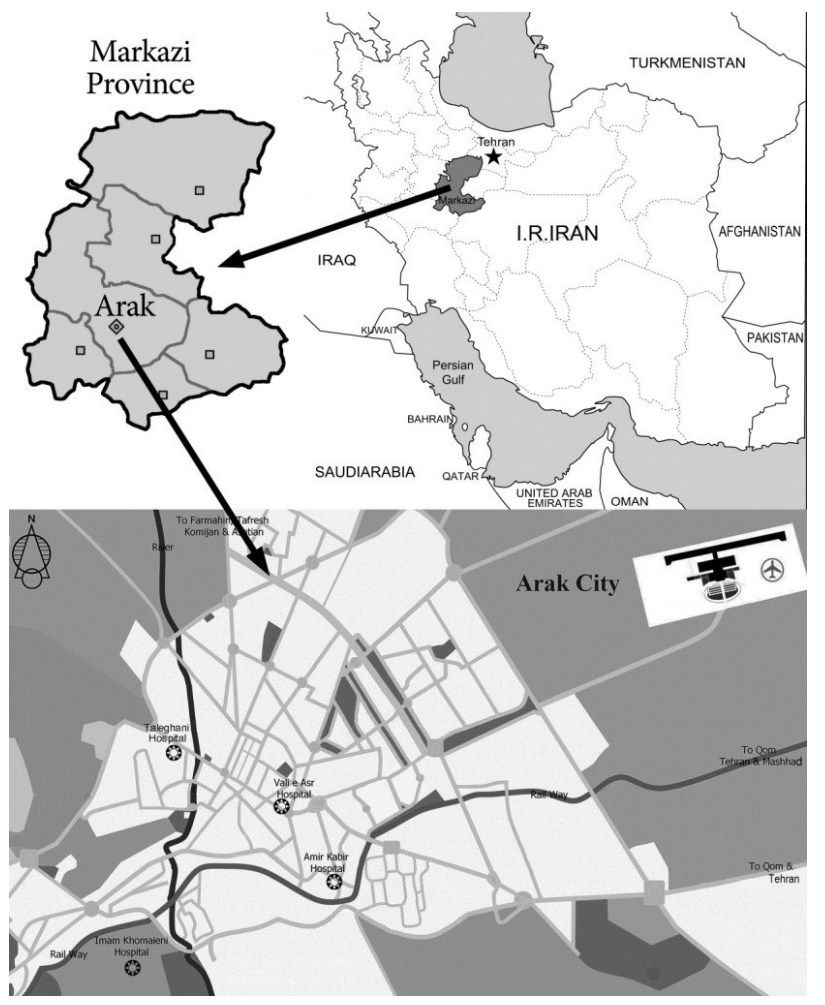

Figure 1. Geographical location of the sampling points in Arak (central area, Iran) where samples of the cooling systems were obtained from excelsior filters. or unit, sample, type and number of the sample was properly recorded. The samples were placed in the sterile plate containing $10 \mathrm{cc}$ Page's amoeba saline. Then samples were shacked for 10 minutes. The fluid was transferred into a sterile $10 \mathrm{~mL}$ conical centrifuge tube, and centrifuged (500 rpm, 10 minutes). After removing the $8 \mathrm{~mL}$ supernatant, the remaining sediment was used in direct observation and culture procedure, respectively.

Non-nutrient agar (NNA) was prepared with Page's amoeba saline. $0.1 \mathrm{~mL}$ Escherichia coli suspension formerly killed was added to media. For killing of E. coli, suspension was boiled on flame. Two milliliters of remaining sediment, which was prepared before, was placed on sterile condition in cultural media and then was incubated in incubator set at the $37^{\circ} \mathrm{C}$. After 4 days, the plates were monitored daily for the outgrowth of Acanthamoeba every 24 hours. This was repeated finally for 7 days. ${ }^{7}$ Slides were prepared with Giemsa staining and observed (1/40).

\section{Identification of the Amoeba}

Based on the characteristics of Acanthamoeba cyst on specific media and isolates from excelsior filters at day 7 (Figure 2). Cysts have been morphologically described; Astronyxids: species having relatively large cysts with smooth ectocyst and stellate endocyst. ${ }^{8}$ The average diameter of cysts was relatively large. They were $<18 \mathrm{mi}-$ cron but the spherical cysts of Naegleria are single-walled and 8-12 micrometers in diameter. ${ }^{9}$ Unlike Acanthamoeba spp., Balamuthia mandrillaris is difficult to isolate and to culture in specific media. The cyst wall of $B$. mandrillaris is made up of a thin, wavy ectocyst, a fibrous mesocyst, and a thick, round endocyst. ${ }^{7}$

The data collected were entered to the Microsoft Excel spread sheet, coded appropriately, and transferred in to SPSS version 20 software package. Data were analysis by descriptive and inference statistical methods, and the

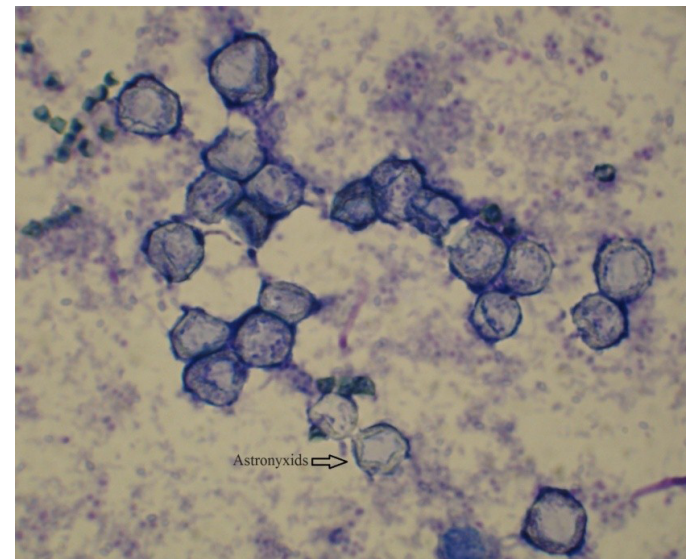

Figure 2. Cysts of Acanthamoeba spp. Growing on Non-nutrient Agar at Day 7: Astronyxids Type. 
Table 1. Number and Percentage of Positive Cases Based on Location and Method of Sampling From Cooling System Filters of Hospitals

\begin{tabular}{lcccccccc}
\hline \multirow{2}{*}{ Hospitals $^{\mathbf{a}}$} & \multicolumn{2}{c}{ No. of Cooling Systems } & \multicolumn{2}{c}{ All Samples } & \multicolumn{2}{c}{ Excelsior Samples } & \multicolumn{3}{c}{ Settled Dust on Excelsior Samples } \\
\cline { 2 - 8 } & Total & No. (\%) & Total & No. (\%) & Total & No. (\%) & Total & No. (\%) \\
\hline A & 5 & $1(25)$ & 10 & $1(10)$ & 5 & $1(25)$ & 5 & $0(0)$ \\
B & 33 & $11(33.3)$ & 66 & $19(28.8)$ & 33 & $11(33.3)$ & 33 & $8(24.2)$ \\
C & 16 & $4(25)$ & 32 & $8(25)$ & 16 & $4(25)$ & 16 & $4(25)$ \\
D & 30 & $7(23.3)$ & 60 & $13(21.7)$ & 30 & $7(23.3)$ & 30 & $6(20)$ \\
Total & 84 & $23(27.3)$ & 168 & $41(24.4)$ & 84 & $23(27.3)$ & 84 & $18(21.4)$ \\
\hline
\end{tabular}

${ }^{a}$ For the ethical and legal reasons, the names of hospitals are alphabetically listed.

statistical significance was considered $P<0.05$.

\section{Results}

Table 1 shows the number of positive cases based on location of sampling. The highest and the lowest rate of contamination were observed in cooling systems of $B$ and $D$ hospitals, respectively. The mean of contamination was $27.3 \%$. Despite the higher percentage of positive excelsior cultivation to culture of settled dust, separated by environmental sampling pump and filter, no significant differences were found between two methods of sampling $(P>0.05)$.

Table 2 shows the positive cases based on wards/units of sampling.

Cooling systems of Ophthalmology (100\%) and Burn (60\%), Neurology (50\%), Surgery (43.8\%) and Infectious Diseases (20\%) and Orthopedic wards as well as intensive care unit (ICU), compared with other wards or units showed higher rates of positive cultures for Acanthamoeba.

\section{Discussion}

The results showed that filters of cooling systems of hospitals in Arak might be contaminated with Acanthamoeba spp. This highlights the potential risk of diffusion of Acanthamoeba cysts originating from excelsior filters of cooling systems into hospital wards. This is the first investigation of the distribution of free-living amoebae (FLA) in excelsior filters of cooling systems of hospital wards in Iran. Several studies have been performed on detection of Acanthamoeba in outdoor and indoor of hospitals in Iran. A previous study in which dust and biofilm samples were collected from various hospital wards of five teaching hospitals in Tehran found 37 out of 70 samples (52.9\%) to be positive for FLA. ${ }^{10}$ In our research 27.3 of excelsior samples were positive whereas 21.4 of samples from settled dust on excelsiors were positive. This difference may be due to transfer of existing Acanthamoeba to the culture medium of excelsior samples.

Many studies on detection of Acanthamoeba in water sources have been carried out. ${ }^{11-13}$ FLA colonizes in dif-
Table 2. Number and Percentage of Positive Cases Based on Wards/Units of Hospitals

\begin{tabular}{lccc}
\hline \multirow{2}{*}{ Wards/Units } & $\begin{array}{c}\text { No. of } \\
\text { Wards/Units }\end{array}$ & \multicolumn{2}{c}{ No. of Cooling Systems } \\
\cline { 3 - 4 } Surgery & 5 & 16 & $7(43.8)$ \\
Burn & 1 & 5 & $3(60)$ \\
Neurology & 1 & 4 & $2(50)$ \\
ENT & 1 & 1 & $0(0)$ \\
CCU & 1 & 1 & $0(0)$ \\
ICU. & 1 & 5 & $1(20)$ \\
NICU & 1 & 1 & $0(0)$ \\
Emergency & 1 & 5 & $0(0)$ \\
Laboratory & 2 & 7 & $1(14.3)$ \\
Maternity & 1 & 2 & $0(0)$ \\
Blood disorders & 1 & 1 & $0(0)$ \\
Ophthalmology & 1 & 1 & $1(100)$ \\
Orthopedic & 2 & 10 & $2(20)$ \\
Infectious diseases & 1 & 5 & $1(20)$ \\
Internal medicine & 2 & 5 & $4(80)$ \\
Delivery & 2 & 9 & $1(11.1)$ \\
Corridors & 2 & 6 & $0(0)$ \\
Total & 26 & 84 & $23(27.3)$ \\
\hline & & & \\
\hline
\end{tabular}

ferent water systems including cooling systems that work with water especially when they are in the open air and are exposed to contaminated dust. ${ }^{3,5,14}$ The cool air contains aerosols and water droplets, which spread in rooms and corridors of hospitals through air ducts, and may carry cysts of Acanthamoeba species. Thus, direct contact with water or inhalation of aerosols in hospital environment may lead to the patients being infected..$^{15,16}$ Infections may occur directly by Acanthamoeba or by various bacteria that they carry. Several studies showed that the free living amoebae that inhabit man-made aquatic systems are partially responsible for the growth and transportation of pathogen bacteria in the same environment. ${ }^{17}$ Intra-amoebic bacteria have been identified with equal frequency in isolates from the environment and from man. Approximate- 
ly 25of such Acanthamoeba isolates carry obligate endosymbionts. ${ }^{18}$ In recent years, despite the already well-documented relationship between Legionella spp. and several species of FLA, an increasing number of gram-negative endosymbionts have been described in amoeba. ${ }^{19}$

The incidence of hospital-acquired achanthamebiasis is not exactly known and most relevant studies have been done on bacterial agents. Nosocomial clusters bacterial infection have been shown to be associated with contaminated cooling towers and potable water supplies, ${ }^{20}$ which may carry Acanthamoeba species. These results thus recommend substitution of aqueous cooling system by non-aqueous or closed circulation cooling systems, especially where susceptible patients receive medical care.

\section{Study Limitation}

Given the limited sample size of this study, caution should be exercised in generalizing our quantitative results. In addition, relative frequency of positive samples in hospital wards is hardy representative, and may vary in different contexts.

\section{Conclusions}

Our results indicate a significant level of amoebae cysts contamination of cooling systems with excelsior filters in various hospital wards. Given the potential contribution of cyst to nosocomial infection, a prompt addressing of the problem is warranted. This may partly be accomplished by using non-aqueous or closed circulation cooling systems. Our study also highlights the need for similar investigations in other health settings and in larger scales to gain insight into the extent of the problem and the associated risks.

\section{Abbreviations}

(FLA): Free-living amoebae; (NNA): non-nutrient agar.

\section{Authors' Contributions}

MM conceived the project idea and designed the study protocol and drafted the paper; SK collected the data and $\mathrm{RH}$ and $\mathrm{BG}$ examined samples and analyzed the data. All authors contributed to the writing of the paper and approved submitted version of the paper.

\section{Competing Interests}

The authors declare no competing interests.

\section{Acknowledgments}

The authors are grateful to Dr. Zahra Eslamirad, DR. Hamid Reza Ahadi and the Parasitology \& Mycology Laboratory of the Arak University of Medical Sciences, hospitals managers and staff for supporting of this study. The Research Deputy of Arak University of Medical Sciences financially supported this research (Project ID 1187).

\section{References}

1. da Rocha-Azevedo B, Tanowitz HB, Marciano-Cabral F. Diagnosis of infections caused by pathogenic free-living amoebae. Interdiscip Perspect Infect Dis. 2009;2009:251406. doi:10.1155/2009/251406.

2. Rodriguez-Zaragoza S. Ecology of free-living amoebae. Crit Rev Microbiol. 1994;20(3):225-241. doi:10.3109/10408419409114556.

3. Berk SG, Gunderson JH, Newsome AL, et al. Occurrence of infected amoebae in cooling towers compared with natural aquatic environments: implications for emerging pathogens. Environ Sci Technol. 2006;40(23):7440-7444.

4. Marciano-Cabral F, Cabral G. Acanthamoeba spp. as Agents of Disease in Humans. Clin Microbiol Rev. 2003;16(2):273307.

5. Cateau E, Delafont V, Hechard Y, Rodier MH. Free-living amoebae: what part do they play in healthcare-associated infections? J Hosp Infect. 2014;87(3):131-140. doi:10.1016/j. jhin.2014.05.001.

6. MalekHosseini A, Maleki A. The effects of climate on traditional architecture and modern city of Arak. Amayesh Geographical Journal. 2010;11:133-155. [Persian].

7. Schuster FL. Cultivation of pathogenic and opportunistic free-living amebas. Clin Microbiol Rev. 2002;15(3):342-354.

8. Pens CJ, da Costa M, Fadanelli C, Caumo K, Rott M. Acanthamoeba spp. and bacterial contamination in contact lens storage cases and the relationship to user profiles. Parasitol Res. 2008;103(6):1241-1245. doi:10.1007/s00436008-1120-3.

9. Markell E, John D, Krotoski W. Markell and Voge's Medical Parasitology. 8th Edition ed. Philadelphia: W.B. Saunders Company; 1999.

10. Lasjerdi Z, Niyyati M, Haghighi A, et al. Potentially pathogenic free-living amoebae isolated from hospital wards with immunodeficient patients in Tehran, Iran. Parasitol Res. 2011;109(3):575-580. doi:10.1007/s00436-011-2288-5.

11. Rezaeian M, Niyyati M, Farnia S, Haghi A. Isolation of Acanthamoeba spp. from Different Environmental Sources. Iran J Parasitol. 2008;3(1):44-47.

12. Mosayebi M, Ghorbanzadeh B, Eslamirad Z, Ejtehadifar M, Rastad B. The Isolation and Detection of Acanthamoeba Keratitis in Rural Water Sources of Arak, Iran. Medical Laboratory Journal. 2014;7(4):66-71.

13. Bagheri $H$, Shafiei $R$, Shafiei $F$, Sajjadi S. Isolation of acanthamoeba spp. From drinking waters in several hospitals of iran. Iran J Parasitol. 2010;5(2):19-25. 
14. Decker BK, Palmore TN. The role of water in healthcareassociated infections. Curr Opin Infect Dis. 2013;26(4):345351. doi:10.1097/QCO.0b013e3283630adf.

15. Freije MR. Formulating a risk reduction strategy for waterborne pathogens in hospital water systems. Am J Infect Control. 2005;33(5):S50-S53. doi:10.1016/j. ajic.2005.04.004.

16. Zeybek Z, Binay AR. Growth ability of gram negative bacteria in free-living amoebae. Exp Parasitol. 2014;145 Suppl:S121-126. doi:10.1016/j.exppara.2014.06.009.

17. Fritsche TR, Gautom RK, Seyedirashti S, Bergeron DL, Lindquist TD. Occurrence of bacterial endosymbionts in Acanthamoeba spp. isolated from corneal and environmental specimens and contact lenses. J Clin Microbiol. 1993;31(5):1122-1126.
18. Winiecka-Krusnell J, Linder E. Bacterial infections of freeliving amoebae. Res Microbiol. 2001;152(7):613-619.

19. Rangel-Frausto MS, Rhomberg $P$, Hollis $R J$, et al. Persistence of Legionella pneumophila in a hospital's water system: a 13-year survey. Infect Control Hosp Epidemiol. 1999;20(12):793-797. doi:10.1086/501586.

20. Sabria M, Yu VL. Hospital-acquired legionellosis: solutions for a preventable infection. Lancet Infect Dis. 2002;2(6):368373.

\section{Please cite this article as:}

Mosayebi M, Hajihossein R, Ghorbanzadeh B, Kalantari S. A risk for nosocomial infection: contamination of hospital air cooling systems by Acanthamoeba spp. Int J Hosp Res. 2016;5(1):17-21. doi:10.15171/ijhr.2016.04. 